\title{
Satellite-based Calibration of the TAIGA-HiSCORE Cerenkov Array by the LIDAR on-board CALIPSO
}

\section{Andrea Porelli ${ }^{a, *}$ on behalf of the TAIGA Collaboration}

(a complete list of authors can be found at the end of the proceedings)

${ }^{a}$ DESY-Zeuthen, Platanenallee 6, 15738, Zeuthen, Germany

E-mail: andrea.porelli@desy.de

The wide-angle Air-Cerenkov array HiSCORE is a major component of the TAIGA facility in the Tunka valley, built for Gamma Astronomy and Cosmic Ray research. HiSCORE will contain 120 stations distributed over a $1 \mathrm{~km}^{2}$ area.

Here, we report a multi-year detection of light flashes in HiSCORE from the LIDAR on-board the Sun-synchronous CALIPSO satellite, obtained in 2015-2021. The $100 \mathrm{~mJ}$ laser $(532 \mathrm{~nm})$ is observed up to distances beyond $10 \mathrm{~km}$ away from the ground light spot. This study complements first observations of a space-based LIDAR, performed in 2015-2017 with the 28 station HiSCORE prototype and the CATS-LIDAR on-board the International Space Station. We present HiSCORE calibration methods developed for the LIDAR events to optimize the pointing resolution of the array; and additionally perform a detailed array performance analysis over 6 years.

The angular resolution for this special class of HiSCORE events - bright flashes, with a plane wave light front - is found to be $\sim 0.05^{\circ}$, which is below the high-energy limit of $\sim 0.1^{\circ}$ for air-showers. The satellite event sample allows to optimize rare event search strategies, like for optical flashes of astrophysical origin. We will also report on scheduled joint LIDAR observation by HiSCORE and TAIGA-IACTs, as well as optical ground instruments - to independently calibrate the absolute array-pointing precision.

$37^{\text {th }}$ International Cosmic Ray Conference (ICRC 2021)

July 12th - 23rd, 2021

Online - Berlin, Germany

\footnotetext{
*Presenter
} 


\section{Introduction}

TAIGA-HiSCORE is a Cherenkov shower-front timing array, and is part of the hybrid TAIGAObservatory (Tunka Advanced Instrument for cosmic ray physics and Gamma Astronomy), under construction in the Tunka valley, $50 \mathrm{~km}$ from Lake Baikal, Russia [1, 2]. TAIGA-HiSCORE is today a $0.7 \mathrm{~km}^{2}$ detector array comprising 89 optical detector stations with $\sim 0.5 \mathrm{~m}^{2}$ sensitive area and $0.6 \mathrm{sr}$ field of view each, to detect ns-duration Cherenkov light flashes from gamma ray showers of energy above $10 \mathrm{TeV}$ [3]. Precision pointing for extensive air shower (EAS) reconstruction is a key to detect astrophysical gamma-ray sources with HiSCORE.

Following the serendipitous detection of the CATS LIDAR on-board the International Space Station (ISS) in 2015-17 [4], we present here a multi year analysis of the CALIPSO LIDAR signal detected by HiSCORE in 2015-2021. Such a signal represents a unique tool to verify, in the absence of strong detected astrophysical sources, the absolute HiSCORE pointing precision and accuracy.

In April 2021, we obtained simultaneous and independent CALIPSO LIDAR detections with the two TAIGA-IACT telescopes. We plan to use the IACT reconstructed direction as a reference to verify and calibrate the HiSCORE array, similarly, but with higher precision, to what was done with the MASTER optical telescope detection of CATS LIDAR in [4].

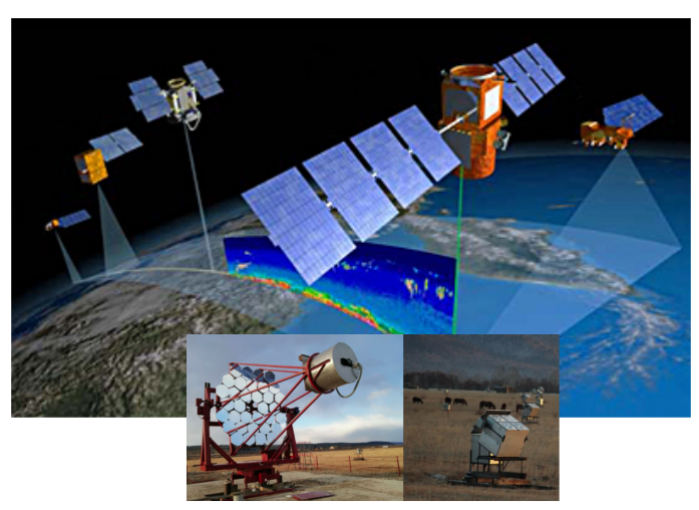

(a)

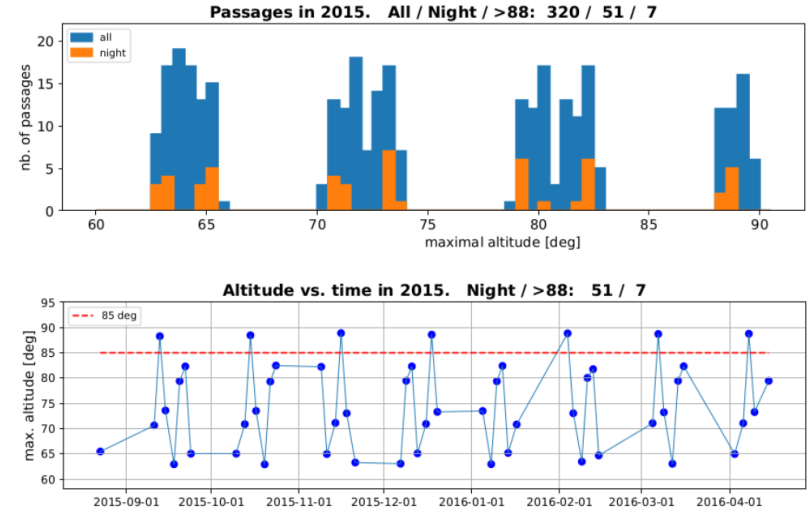

(b)

Figure 1: CALIPSO passage above TAIGA site. (a) Artistic view of CALIPSO orbiting around Earth as part of the "A-Train" satellite constellation. On the bottom, a TAIGA-IACT (left) and a TAIGA-HiSCORE station (right) "receiving" light from the CALIPSO LIDAR. (b) Predicted CALIPSO passages above TAIGA during season 2015/16: satellite passages maximum altitude distribution (top), and as a function of date (bottom). Statistics is given for all days and only-dark-nights. Only passages above $88^{\circ}$ have been observed by HiSCORE.

\section{TAIGA detection of the LIDAR onboard the CALIPSO satellite}

\subsection{CALIPSO}

The CALIPSO (Cloud Aerosol Lidar and Infrared Pathfinder Satellite Observations) satellite was launched on April 28, 2006 as a joint NASA and CNES mission [12], as part of a seven satellite 
constellation known as the "A-Train" (see figure 1(a)) in Sun-synchronous orbit at an altitude of approximately $700 \mathrm{~km}$. With an orbit every 98.4 minutes ( 14.6 orbits per day), it repeats its track every 16 days. CALIPSO contains a LIDAR known as CALIOP (Cloud Aerosol Lidar with Orthogonal Polarization), with the goal of obtaining high resolution vertical profiles of clouds and aerosol in the Earth's atmosphere. CALIOP produces simultaneous, co-aligned $20 \mathrm{~ns}$ pulses (532 and $1064 \mathrm{~nm}$ wave length, $110 \mathrm{~mJ}$ energy), pointed at $3^{\circ}$ away from the geodetic nadir in the forward direction with respect the satellite motion, at a repetition rate of $20.16 \mathrm{~Hz}$. A spot on the ground of approximately $70 \mathrm{~m} \mathrm{rms}$ diameter is estimated. On September 18, 2018, CALIPSO joined the C-Train orbit ( $\sim 16.6 \mathrm{~km}$ below the A-Train), together with the CloudSat spacecraft.

To predict date, time, and the maximum altitude of CALIPSO passages over TAIGA we used the PyEphem package [10]. Figure 1(b) shows the predicted maximum altitude during season 2015/16. The full satellite track in the sky is obtained using the SGP4 propagator together with CALIPSO historical TLE [11].
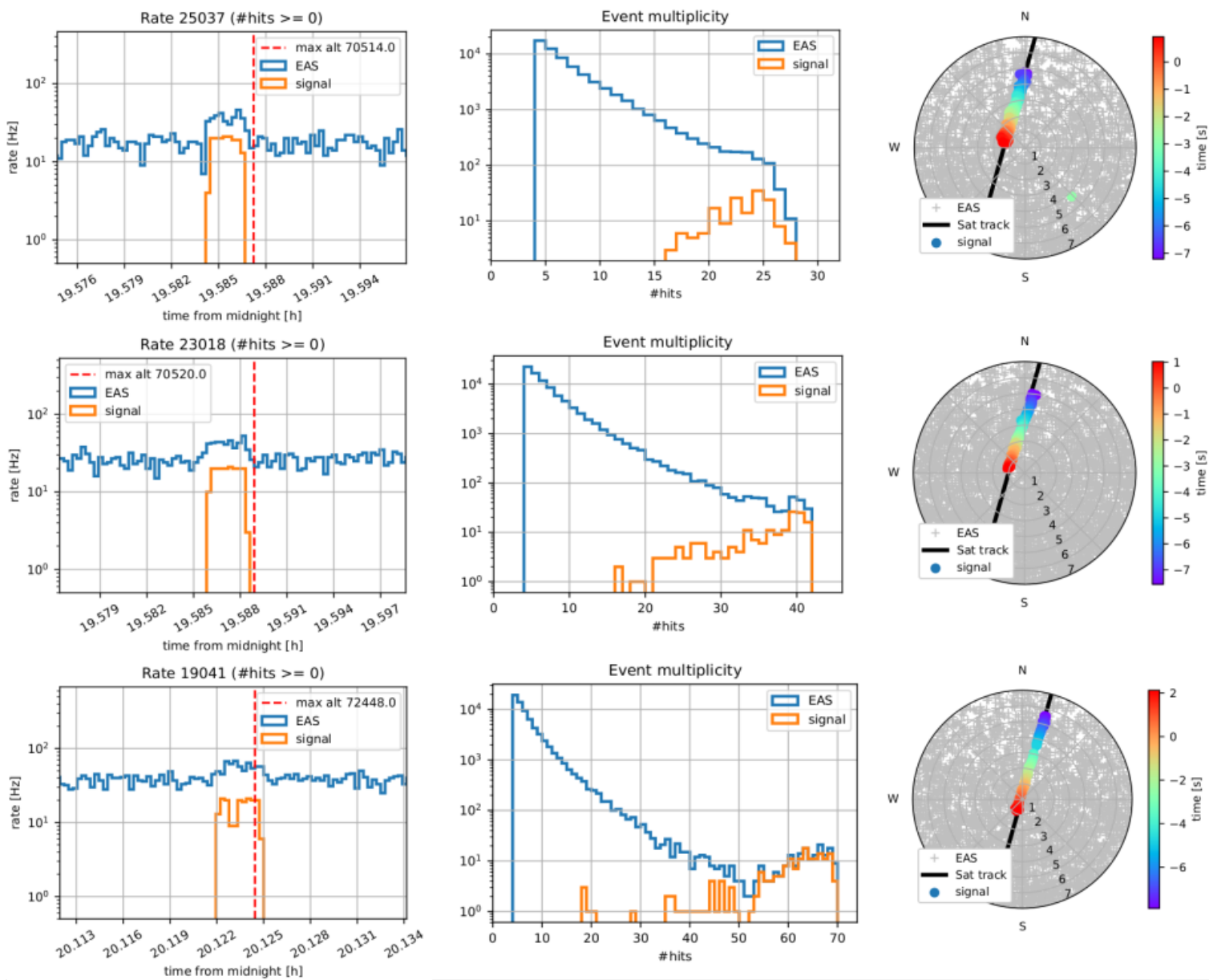

Figure 2: TAIGA-HiSCORE "SAT" (orange) and "EAS" (blue) detected events. Left: event rate; middle: event multiplicity; right: reconstructed direction and time (color coded) evolution. Top: Run 25/03/2017 (1 cluster array). Middle: Run 23/01/2018 ( 2 cluster array). Bottom: Run 19/04/2021 (3 cluster array). 


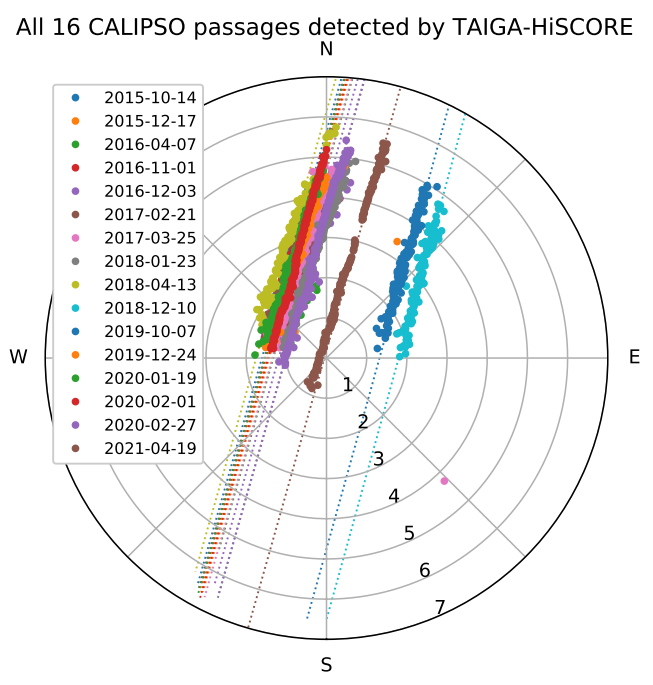

(a)

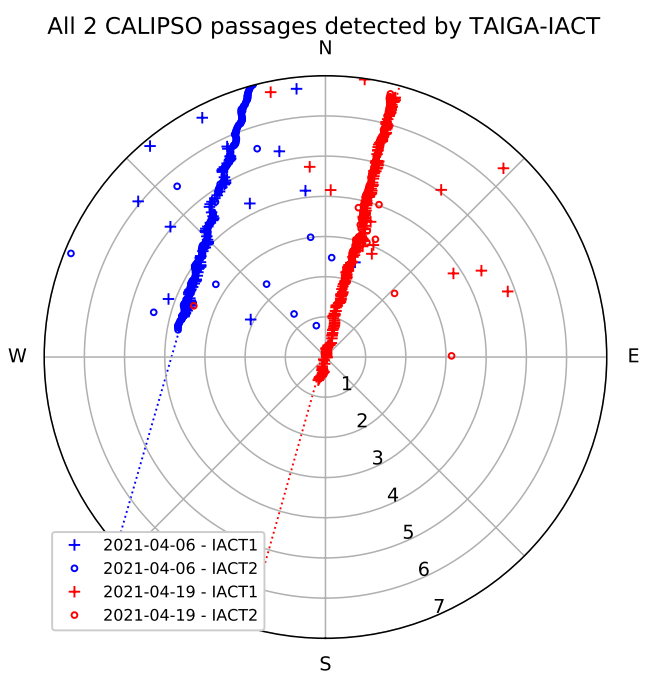

(b)

Figure 3: Compilation of detected CALIPSO passages over the TAIGA Observatory. (a) HiSCORE: 16 reconstructions of CALIPSO from 2015-2021. (b) IACT: 2 passages in both IACTs (4 reconstructions) of CALIPSO from the April 2021 observation campaign. The satellite tracks are obtained using the SGP4 propagator together with public TLEs.

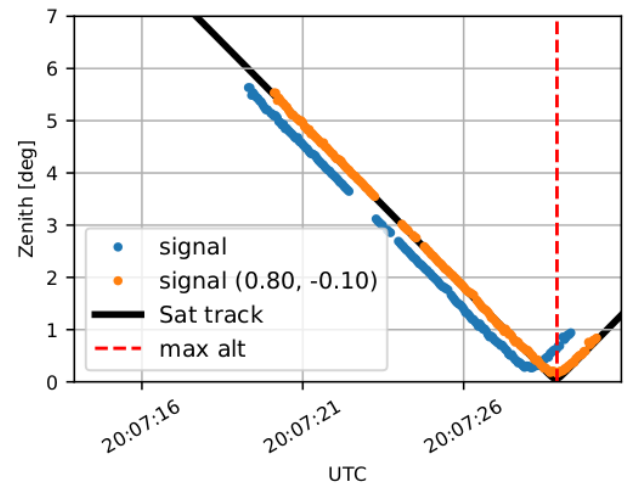

(a)

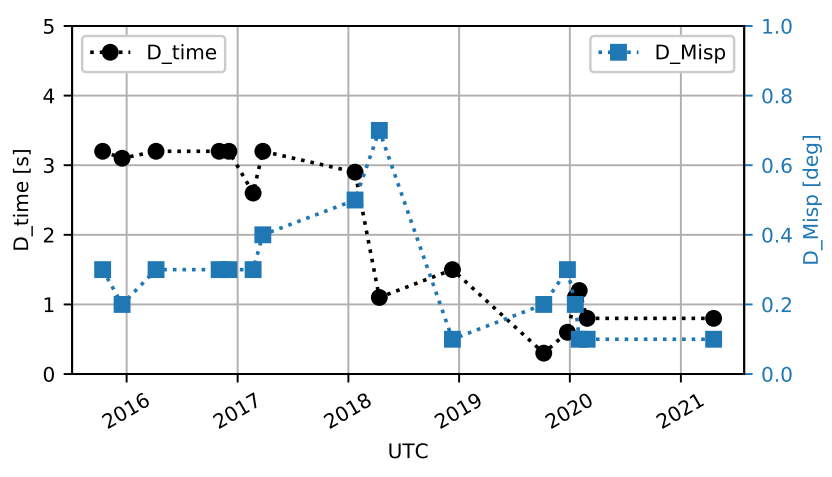

(b)

Figure 4: Preliminary "raw" CALIPSO-HiSCORE calibration. (a) reconstructed Zenith vs time, for passage 19/04/2021. Blue: original reconstruction; Orange: reconstruction after raw calibration (D_time $=0.8 \mathrm{~s}$, D_Misp $=0.1^{\circ}$, see text for details). (b) D_time, and D_Misp "raw" corrections as function of passage time.

\subsection{TAIGA-HiSCORE}

The reconstruction of those HiSCORE events that are due to laser pulses from the satellite ("SAT events") is obtained with the HiSCORE standard data analysis procedure [3, 7]. For this work, the Hybrid array time calibration is used [6]. This type of events differs from normal EAS events for their arrival time front, well approximated by a plane wave (first step of EAS reconstruction). Knowing the time of the satellite maximal altitude, $t_{\max }$, the satellite speed, and the laser beam pointing direction, it is possible to isolate the SAT events by selecting a time interval of $[-15,+5] \mathrm{s}$ around $t_{\text {max }}$. A further 


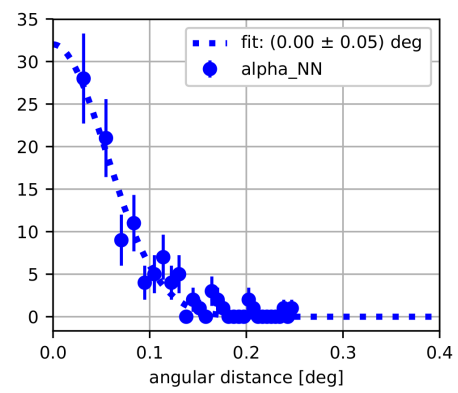

Figure 5: Pointing resolution for HiSCORE SAT laser events . Distribution of angular distance between consecutive reconstructed directions, divided by $\sqrt{2}$, for passage $10 / 12 / 2018$. Bins scale with $1 / \alpha$. The preliminary upper limit for the angular error is $0.05^{\circ}$ (satellite motion not considered).

selection based on the number of hits $(\geq 15)$, the reconstructed zenith angle $\left(\leq 10^{\circ}\right)$, and a $\chi^{2}$-cut on the plane wave reconstruction, allows to eliminate the remaining EAS from the event sample. Examples of detected and reconstructed passages are given in figure 2 for the 1-, 2- and 3-cluster array configurations of HiSCORE in 2017, 2018 and 2021. Figure 3(a) shows a sky plot (AltAz) containing all the 16 CALISPO passages detected and reconstructed with HiSCORE.

We perform a preliminary verification of the HiSCORE reconstruction (absolute pointing), using as reference the predicted satellite track. It must be noted that the satellite track propagator (time, location) contains systematic effects still under investigation, thus these results are preliminary, and are shown only to illustrate the calibration concept and its main results. Figure 4(a) gives an example of the preliminary calibration, showing the evolution in time of the reconstructed zenith angle, and compares with the absolute track prediction (black line). By minimizing the space angle between the two tracks, two parameters are extracted: a time shift, D_theta, and the angular distance from the predicted track, D_Misp. We emphasize, that D_Misp is not the real mispointing error of HiSCORE, but a convolution of likely satellite dominated effects as mentioned before. Figure 4(b) shows the distribution of these parameter as function of the passage date, over the 2015-2021 period.

We use SAT events to characterize the directional reconstruction precision of HiSCORE. The pointing precision of plane wave events reconstruction is estimated by the space angle $\alpha_{N N}$, i.e. the space angle between successive laser events (separated in time and direction by $50 \mathrm{~ms}$ and $0.03 \mathrm{deg}$, respectively) divided by $\sqrt{2}$. Figure 5 shows $\alpha_{N N}$ distribution for all events for the passage 10/12/2018, with an rms of $0.05 \mathrm{deg}$. This is better than the pointing precision of $0.1 \mathrm{deg}$ for highest energy EAS [2]. An improved method, based on a precision satellite tracking, is in preparation, and will allow to check for other systematic tracking errors.

\subsection{TAIGA-IACTs}

The discovery and analysis of CALIPSO LIDAR signals in the HiSCORE archival data triggered a targeted observation campaign with the TAIGA-IACTs in April, 2021 (first hints for a satellite passage, see [5]). With the IACTs, we obtained two stereo-mode detections of CALIPSO on April 6 and 19, 2021 , which are displayed in figure 3(b). Their maximum elevations were $86.3^{\circ}$ and $89.9^{\circ}$, which 

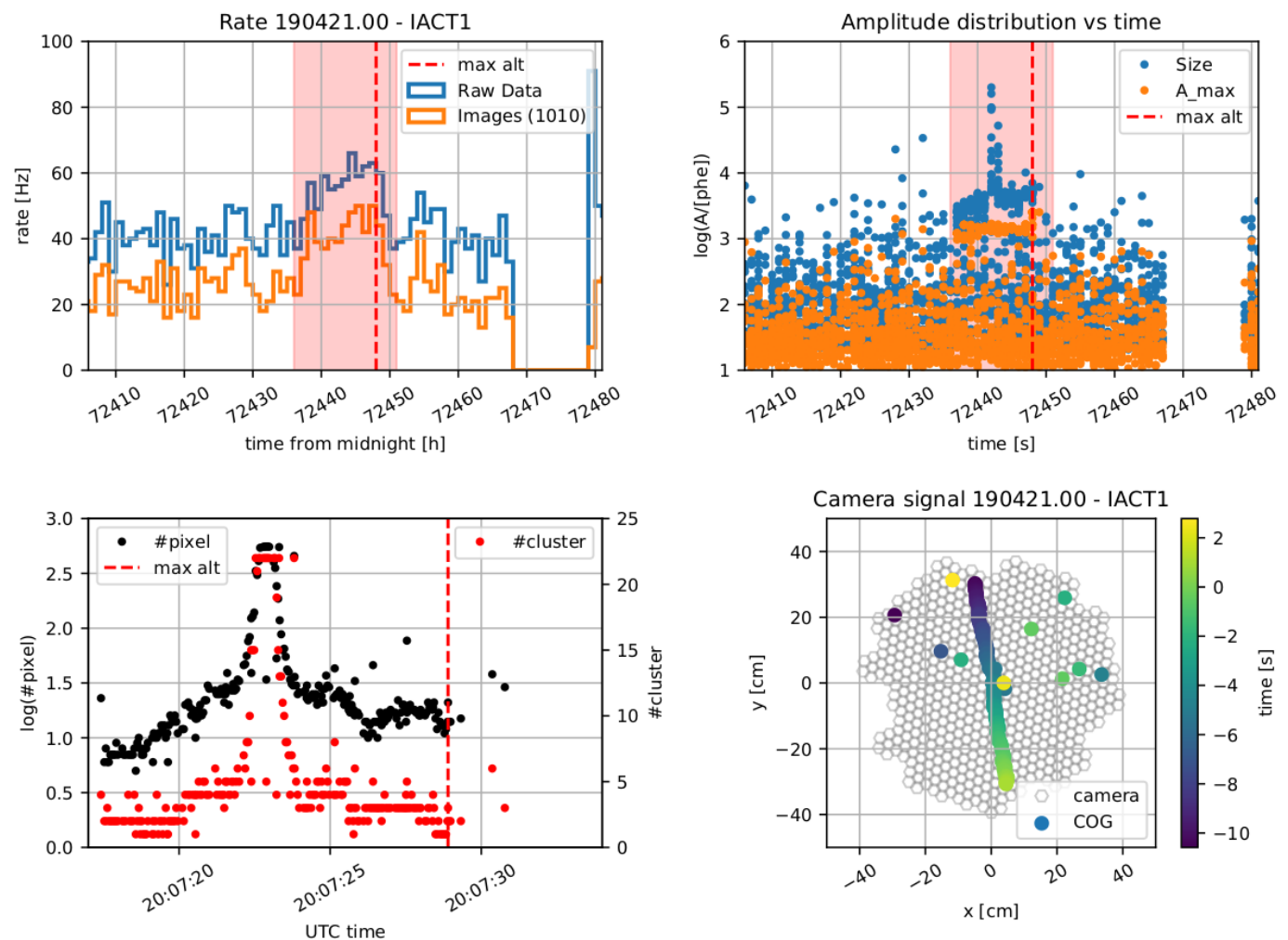

Figure 6: TAIGA-IACT "SAT" event detection. Top-left: trigger rate for raw (blue) and cleaned (orange) camera images, around the passage time. Top-right: image size (blue) and maximum amplitude (orange) vs time, around the passage time. Bottom-left: image cluster (black) and pixel (red) multiplicity for events inside the selection time window (red band in the top row). Bottom-right: camera image center of gravity as function of time, reproducing the signal track.

corresponds to a laser foot print distance to the observatory of $\sim 45 \mathrm{~km}$ and $\sim 1 \mathrm{~km}$, respectively. These are the first space-based LIDAR detections with IACT telescopes on ground.

Figure 6 shows the 19/04/2021 CALIPSO passage detected by TAIGA-IACT telescope 1 . This is the first ever combined IACT-HiSCORE LIDAR detection. The top-left panel shows the camera trigger rate for raw (blue) and cleaned (orange) images, around $t_{\max }$ (red dashed line); the image cleaning selects pixels with amplitude larger than 10 phe. The top-right panel shows, for the same time interval, the image size and the maximum amplitude distributions. To separate SAT from EAS induced images, a simple event selection based on event time ( $t_{S A T} \in[-13,+3] \mathrm{s}$ around $t_{\text {max }}$, red band) and max amplitude $\left(A_{\max } \geq 300\right.$ phe) is performed. The bottom-left panel shows the image cluster (red) and pixel (black) multiplicity for the selected SAT events. The reconstruction of the signal arrival direction is obtained by calculating the pixel amplitude center of gravity (COG) inside the camera, and then projecting it into the local AltAz sky (see figure 3(b)). The bottom-right panel of figure 6 shows the image COG time evolution inside the camera, reproducing a clear track. Analysis of this particular passage is difficult, due to serious saturation effects in the camera (close-by passage).

The main goal of a combined IACT and HiSCORE detection of the LIDAR is, to use the 
satellite direction directly measured with the TAIGA-IACT as an independent and precise localization of the emission point, and thus solving the systematic uncertainties related to precision satellite track predictions discussed above for the HiSCORE stand-alone LIDAR signal analysis. The satellite localization for each laser pulse thus turns into an absolute reference for HiSCORE - to allow verification of reconstruction, and calibrate the absolute pointing correction. Moreover, the precise knowledge of the emission point allows to directly perform the array time calibration, as done in [6]. This final analysis is still in progress, and will profit from further combined satellite observations, at moderate camera illumination.

\section{Conclusion}

The TAIGA Observatory pioneered observation of space based LIDARs by ground based Air Cerenkov installations: by detecting CATS on board the ISS in 2015-17 with HiSCORE, and now with the 100 times brighter LIDAR on the CALIPSO satellite. First detections of an orbiting laser with IACTs started at TAIGA in April, 2021, including a first combined HiSCORE-IACT observation.

Orbiting LIDARs are now established as point-like sources of laser light from large distance, and generate at ground a nearly plane wave - despite passing up to several $100 \mathrm{~km}$ of air. They are detected also for directions far outside the nominal narrow beam geometry, i.e. for HiSCORE up to elevations of $88^{\circ}$ - up to $10^{\prime} \mathrm{s} \mathrm{km}$ off the footprint. Precise understanding of this emission is currently lacking.

We summarize the main interest of large Cerenkov detectors in space based LIDARs:

- Precision calibration of extended, complex air Cerenkov instruments

- Internal timing offsets between detectors and sub-systems in large setups

- spatial reconstruction verification (pointing, system test) with varying illumination levels

- priority: calibrate absolute pointing of detectors like HiSCORE

- efficient cross comparison and calibration of components in large installations

- Characterization of setups for exotic, short pulsed optical SETI-like signals

- In-flight characterization of the spatial LIDAR beam profile - instrinsically impossible at ground

- Light propagation in atmosphere: unique setup with $700 \mathrm{~km}$ base-length to measures LIDAR forward scattering (amplitude, time delay patterns at various angles)

We note, that the Cerenkov telescope VERITAS had first LIDAR detections in May, 2021, is now scheduling regular observations, and found archival detections up to $54^{\circ}$ (500 km distance) [9].

For the upcoming CTA project, space based LIDARs will be a relevant verification tool.

Outlook for TAIGA:

- obtain absolute pointing, by simultaneous LIDAR detection with IACT and HiSCORE

- improve the HiSCORE plane-wave pointing error assessment

- start routinely hybrid CALIPSO observations by IACT and HiSCORE 


\section{Acknowledgments}

This work was performed at the UNU "Astrophysical Complex of MSU-ISU" (agreement 13.UNN.21.0007). The work is supported by Russian Foundation for Basic Research (grants 19-52-44002, 19-32-60003) the Russian Science Foundation (grant 19-72-20067 (Section 3,4) the Russian Federation Ministry of Science and High Education (projects FZZE-2020-0017, FZZE-2020-0024).

\section{References}

[1] N. Budnev et al, Nuclear Inst. and Methods in Physics Research, A 958 (2020) 162113

[2] N. Budnev et al, PoS(ICRC2021)731

[3] L. Kuzmichev et al, Nuclear Inst. and Methods in Physics Research, A 952 (2020) 161830

[4] A. Porelli et al, PoS(ICRC2017)754

[5] A. Panov, et al , PoS(ICRC2021)951

[6] A. Porelli et al., PoS(ICRC2017)752

[7] A. Porelli et al, PoS(ICRC2021)877

[8] A. Porelli, PhD thesis, Humboldt University (2020), http://edoc.hu-berlin.de/18452/22369

[9] G. Foote et al., 238 AAS meeting (2021)

[10] B. C. Rhodes, (2011), https://ui.adsabs.harvard.edu/abs/2011ascl.soft12014R

[11] F.R. Hoots and R.L. Roehrich, https://www.celestrak.com/columns/v04n03/ (1980)

[12] D. Winker et al, Journal of Atmospheric and Oceanic Technology, Vol. 26, Issue 11, 2310 (2009) 


\section{Full Authors List: TAIGA Collaboration}

I. I. Astapov ${ }^{2}$, A. K. Awad ${ }^{10}$, P. A. Bezyazeekov ${ }^{3}$, M. Blank ${ }^{10}$, E. A. Bonvech ${ }^{1}$, A. N. Borodin ${ }^{4}$, A. V. Bulan ${ }^{1}$, M. Brueckner ${ }^{5}$, N. M. Budnev $^{3}$, A. Chiavassa ${ }^{6}$, D. V. Chernov ${ }^{1}$, A. N. Dyachok ${ }^{3}$, A. R. Gafarov ${ }^{3}$, A. Yu. Garmash ${ }^{7,8}$, V. M. Grebenyuk ${ }^{4,9}$, O. A. Gress ${ }^{3}$, E. Gress $^{3}$, T. I. Gress ${ }^{3}$, O. G. Grishin ${ }^{3}$, A. A. Grinyuk ${ }^{4}$, D. Horns ${ }^{10}$, A. Igoshin ${ }^{1}$, A. D. Ivanova ${ }^{3}$, A. L. Ivanova ${ }^{3,7}$, N. N. Kalmykov ${ }^{1}$, V. V. Kindin $^{2}$, S. N. Kiryuhin ${ }^{3}$, R. P. Kokoulin ${ }^{2}$, K. G. Kompaniets ${ }^{2}$, E. E. Korosteleva ${ }^{1}$, V. A. Kozhin ${ }^{1}$, E. A. Kravchenko ${ }^{7}, 8$, A. P. Kryukov ${ }^{1}$, L. A. Kuzmichev ${ }^{1}$, A. A. Lagutin ${ }^{11}$, M. Lavrova ${ }^{4}$, Yu. E. Lemeshev ${ }^{3}$, B. K. Lubsandorzhiev ${ }^{12}$, N. B. Lubsandorzhiev ${ }^{1}$, A. D. Lukanov ${ }^{12}$, D. S. Lukyantsev ${ }^{3}$, S. D. Malakhov ${ }^{3}$, R. R. Mirgazov $^{3}$, R. Mirzoyan ${ }^{13,3}$, R. D. Monkhoev ${ }^{3}$, E. A. Osipova ${ }^{1}$, A. L. Pakhorukov ${ }^{3}$, A. Pan ${ }^{4}$, L. Panasenko $^{7}$, L. V. Pankov ${ }^{3}$, A. D. Panov ${ }^{1}$, A. A. Petrukhin ${ }^{2}$, I. A. Poddubny ${ }^{3}$, D. A. Podgrudkov ${ }^{1}$, V. A. Poleschuk ${ }^{3}$, V. Ponomareva ${ }^{3}$, M. Popesku $^{14}$, E. G. Popova ${ }^{1}$, A. Porelli ${ }^{5}$, E. B. Postnikov ${ }^{1}$, V. V. Prosinn ${ }^{1}$, V. S. Ptuskin ${ }^{15}$, A. A. Pushnin ${ }^{3}$, R. I. Raikin ${ }^{11}$, A. Y. Razumov ${ }^{1}$, G. I. Rubtsov ${ }^{12}$, E. V. Ryabov ${ }^{3}$, Y. I. Sagan ${ }^{4}, 9$, V. S. Samoliga ${ }^{3}$, A. A. Silaev ${ }^{1}$, A. A. Silaev(junior) ${ }^{1}$, A. Yu. Sidorenkov ${ }^{12}$, A. V. Skurikhin ${ }^{1}$, M. Slunecka ${ }^{4}$, A. V. Sokolov ${ }^{7,8}$, L. G. Sveshnikova ${ }^{1}$, V. A. Tabolenko ${ }^{3}$, A. B. Tanaev ${ }^{3}$, B. A. Tarashansky ${ }^{3}$, M. Yu. Ternovoy ${ }^{3}$, L. G. Tkachev ${ }^{4,9}$, R. Togoo $^{16}$, M. Tluczykont ${ }^{10}$, N. Ushakov ${ }^{12}$, A. Vaidyanathan ${ }^{7}$, P. A. Volchugov ${ }^{1}$, N. V. Volkov ${ }^{11}$, D. Voronin ${ }^{12}$, R. Wischnewski $^{5}$, A. V. Zagorodnikov ${ }^{3}$, D. P. Zhurov ${ }^{3,17}$, I. I. Yashin ${ }^{2}$

${ }^{1}$ Skobeltsyn Institute of Nuclear Physics, Moscow State University, Moscow, 119991 Russia. ${ }^{2}$ National Research Nuclear University MEPhI, Moscow, 115409 Russia. ${ }^{3}$ Research Institute of Applied Physics, Irkutsk State University, Irkutsk, 664003 Russia. ${ }^{4}$ Joint Institute for Nuclear Research, Dubna, Moscow oblast, 141980 Russia. ${ }^{5}$ Deutsches Elektronen-Synchrotron DESY, Zeuthen, 15738 Germany. ${ }^{6}$ Physics Department of the University of Torino and Istituto Nazionale di Fisica Nucleare, Torino, 10125 Italy. ${ }^{7}$ Novosibirsk State University, Novosibirsk, 630090 Russia. ${ }^{8}$ Budker Institute of Nuclear Physics, Siberian Branch, Russian Academy of Sciences, Novosibirsk, 630090 Russia. ${ }^{9}$ Dubna University, Dubna, Moscow oblast, 141980 Russia. ${ }^{10}$ Institut für Experimentalphysik, Universität Hamburg, Hamburg, D-22 761 Germany. ${ }^{11}$ Altai State University, Barnaul, Altai krai, 656049 Russia. ${ }^{12}$ Institute for Nuclear Research, Russian Academy of Sciences, Moscow, 117312 Russia. ${ }^{13}$ Max Planck Institute for Physics, Munich, 80805 Germany. ${ }^{14}$ Space Science Institute, Magurele, 077125 Romania. ${ }^{15}$ Pushkov Institute of Terrestrial Magnetism, Ionosphere and Radio Wave Propagation, Russian Academy of Sciences, Troitsk, Moscow, 142190 Russia. ${ }^{16}$ Institute of Physics and Technology Mongolian Academy of Sciences, Ulaanbaatar, Mongolia. ${ }^{17}$ Irkutsk National Research Technical University, Irkutsk, Russia. 